\title{
A critical role for the co-repressor N-CoR in erythroid differentiation and heme synthesis
}

\author{
Dianzheng Zhang ${ }^{1}$, Ellen Cho ${ }^{1}$, Jiemin Wong ${ }^{2,3}$ \\ ${ }^{I}$ Department of Biochemistry and Molecular Biology, Philadelphia College of Osteopathic Medicine, 4710 City Avenue, Philadelphia, \\ PA 19131-1694, USA; '2Department of Molecular and Cellular Biology, Baylor College of Medicine, One Baylor Plaza, Houston, \\ TX 77030, USA
}

Co-repressor N-CoR (nuclear receptor co-repressor) has important roles in different biological processes, including proliferation, differentiation and development. Mutant mice lacking N-CoR are embryonically lethal and appear to die from anemia owing to defects in definitive erythropoiesis. However, the underlying molecular mechanisms of N-CoRmediated erythroid differentiation are largely unknown. Using the human erythroleukemic K562 cell line, which can be chemically induced to differentiate into either erythroid or megakaryocytic lineages depending on the inducers used, we have investigated the role of N-CoR in erythroid differentiation. We show that knockdown of N-CoR either transiently (siRNA) or permanently (shRNA) impairs the cytosine arabinoside (Ara-C)- but not hemin-induced erythroid differentiation of K562 cells. RT-PCR analysis reveals that N-CoR is required for induction by Ara-C of 5-aminolevulinate synthase (ALA-S2), a key enzyme involved in heme biosynthesis. Furthermore, the amount of N-CoR proteins increases significantly during Ara-C-induced K562 differentiation, apparently through a post-transcriptional mechanism. Consistent with the data from N-CoR-null mice, N-CoR is not required for the differentiation of K562 cells into megakaryocytic lineages, induced by phorbol 12-myristate 13-acetate. Thus, our in vitro study confirms a role for N-CoR in erythroid differentiation and reveals for the first time that N-CoR is required for the induction of a key enzyme involved in heme synthesis.

Keywords: co-repressor N-CoR, K562, erythroid differentiation, heme, hemin, Ara-C

Cell Research (2007) 17:804-814. doi: 10.1038/cr.2007.72; published online 4 September 2007

\section{Introduction}

Transcriptional regulation is one of the major steps in controlling cell differentiation, and transcriptional co-activators and co-repressors have pivotal roles in this process $[1,2]$. N-CoR (nuclear receptor corepressor) and SMRT (silencing mediator for retinoid and thyroid hormone receptor) are two highly related proteins that were originally

\footnotetext{
Correspondence: Jiemin Wong

Tel: +86-21-54344030

E-mail: jmweng@bio.ecnu.edu.cn

${ }^{3}$ Current address: Institute of Biomedical Sciences, College of Life Science, East China Normal University, Dongchuan Road 500, Shanghai 200241, China

Received 7 January 2007; revised 28 April 2007; accepted 10 May 2007; published online 4 September 2007
}

isolated and characterized on the basis of their abilities to interact with and mediate repression by unliganded forms of thyroid hormone and retinoic acid receptors [3, 4]. Recent studies demonstrate that $\mathrm{N}-\mathrm{CoR}$ and SMRT have important roles in multiple biological processes, including proliferation, differentiation, apoptosis and development [1, 5-10]. For example, homozygous deletion of $\mathrm{N}-\mathrm{CoR}$ in mice is embryonic lethal, and N-CoR-null mice show defects in neural cell differentiation and developmental progression of specific erythrocytes and thymocytes [5]. N-CoR is required for definitive rather than primitive erythropoiesis [5], since peripheral blood smears from $\mathrm{N}-\mathrm{CoR}$-/- embryos showed fewer non-nucleated erythrocytes but many more nucleated erythroblasts than wild-type controls. Significantly, the severe anemia appears to be the cause of the embryonic lethality of N-CoR-null mice. However, N$\mathrm{CoR}$ is not required for the generation of megakaryocytes 
because a similar number of megakaryocytes were found in wild type and N-CoR-/- fetal livers [5]. How N-CoR regulates definitive erythropoiesis is not yet clear.

The K562 cell line was established more than 30 years ago [11] from a patient with an acute transformation of chronic myelogenous leukemia. K562 cells are multipotent stem cells and can differentiate into erythroid lineages upon induction with different chemicals, including hemin, cytosine arabinoside (Ara-C) and 5-azacytidine [12-15]. As a canonical agent for erythroid differentiation, hemin exerts a profound effect on K562 cell maturation and promotes the synthesis of the fetal and embryonic hemoglobins [16]. Ara-C is a deoxycytidine analog and is one of the most commonly used drugs in leukemia therapy [17]. Once it enters the cell, Ara-C is phosphorylated and converted to its active form, cytosine arabinoside triphosphate (Ara-CTP). Ara-CTP competes with dCTP for incorporation into DNA during DNA replication, and, once incorporated, Ara-C disrupts further DNA synthesis and results in the initiation of the downstream cellular response to DNA damage [18, 19]. K562 cells are resistant to low doses of Ara-C in terms of apoptosis. Instead, low doses of Ara-C induce K562 cell erythroid differentiation [13]. Although how Ara-C induces K562 differentiation toward the erythroid lineage remains unclear, microarray data from Ara-C-induced K562 cells show that systemic upregulation of genes is involved in erythroid differentiation [17].

In this study, we used chemically induced K562 cell erythroid differentiation as a model system to investigate the role for $\mathrm{N}-\mathrm{CoR}$ in erythroid differentiation. We show that $\mathrm{N}-\mathrm{CoR}$ is required for Ara-C- but not hemin-induced erythroid differentiation. Knockdown of N-CoR impaired the induction of a key enzyme, 5-aminolevulinate synthase (ALA-S2), that is involved in heme biosynthesis. Consistent with the phenotype of the N-CoR-/- mice, we found that N-CoR is not essential for the differentiation of K562 cells into megakaryocytes.

\section{Materials and Methods}

\section{Cell culture and chemical treatment}

K562 cells, kindly provided by Dr Chan (Department of Pharmacology, Baylor College of Medicine), were grown on RPMI 1640 (with $300 \mathrm{mg} / \mathrm{l}$ glutamine) supplemented with $10 \%$ fetal bovine serum and $1 \times$ antibiotics and antimycotics $(100 \mathrm{U} / \mathrm{ml}$ ampicillin, $100 \mathrm{U} / \mathrm{ml}$ streptomycin and $0.25 \mu \mathrm{g} / \mathrm{ml}$ amphotericin) at $37^{\circ} \mathrm{C}$ in a $5 \% \mathrm{CO}_{2}$ incubator. In the experiments, the cells were seeded at a density of $2 \times 10^{6}$ cells $/ 10 \mathrm{ml}$ in $100-\mathrm{mm}$ dishes and cultured for 3 to 6 days in the presence or absence of the inducing agents. The inducing reagents Ara-C, hemin, phorbol 12-myristate 13-acetate (PMA) and the benzidine-staining reagents were purchased from Sigma.

\section{Nuclear extract and western blot}

Chemically treated or control cells were collected and washed with cold PBS twice. Cells were centrifuged for $5 \mathrm{~min}$ at 3000 r.p.m. Cells were lysed in $1 \mathrm{ml}$ of a lysis buffer (10 mM Tris-HCl, $\mathrm{pH} 7.4,10$ $\mathrm{mM} \mathrm{NaCl}, 3 \mathrm{mM} \mathrm{MgCl}, 0.5 \% \mathrm{NP}-40$ ) with freshly added protease inhibitors. Cell lysate was incubated for $5 \mathrm{~min}$ on ice and centrifuged at 4000 r.p.m. for $5 \mathrm{~min}$. The supernatant was removed and the nuclei were re-suspended into $100 \mu \mathrm{l}$ of an extraction buffer $(20 \mathrm{mM}$ Tris-HCl, pH 7.9, 420 mM KCl, 0.2 mM EDTA, 10\% glycerol, 2 mM DTT) with freshly added protease inhibitors, and then vortexed vigorously for 1 to $2 \mathrm{~min}$ and incubated on ice for $30 \mathrm{~min}$. Nuclear lysate was centrifuged for $20 \mathrm{~min}$ at 14000 r.p.m. Supernatant was collected as nuclear extract and an equal amount of total proteins was separated by $7.5 \%$ SDS-PAGE. Western blotting was carried out routinely with specific antibodies as described [20].

\section{RNAi and shRNA}

The small interference RNA (siRNA) against N-CoR was as described [8]. For transient knockdown of mRNA, K562 cells were washed twice with PBS and re-suspended in the reduced serum medium OPTI MEM (Invitrogen) at the concentration of $1 \times 10^{7}$ cells $/ \mathrm{ml}$. For electroporation, $0.2 \mathrm{ml}$ of cells were mixed with $7 \mu \mathrm{l}$ of $20 \mu \mathrm{M}$ siRNA and incubated for $5 \mathrm{~min}$ at room temperature. The electroporation was conducted using the BioRad electroporator at the setting of $300 \mathrm{mV} / 125 \mu \mathrm{F}$. Cells were seeded in $10 \mathrm{ml}$ of culture medium and cultured for 3 days before collection for subsequent analysis. For the rescue experiments, a mouse N-CoR expression plasmid (pCMX-mN-CoR) was transfected by electroporation at the same setting mentioned above. To make a stable cell line with shN-CoR, a specific 64-nt oligonucleotide DNA fragment was cloned in the $\mathrm{B} g / \mathrm{II} / \mathrm{Hid}$ III sites of the pSUPER.retro vector (Oligoengine). Within the 64-nt oligonucleotide, the 19-nt target (GCTACTTCTCGAGGAAACA) is included in both the sense and antisense orientation, separated by a 9-nt spacer sequence. Plasmid DNA containing the designed insert was transfected to K562 cells by electroporation at $300 \mathrm{mV} / 125 \mu \mathrm{F}$. Puromycin was used for selecting stably transfected cells as instructed by the manufacturer.

\section{Benzidine staining of erythroid cells}

Benzidine staining was used to detect the pseudoperoxidase activity of hemoglobin in K562 cells and performed as described [21]. K562 cells were treated with or without inducing agents for 3 to 6 days. The benzidine solution contained $0.2 \%$ 3,3'-dimethoxybenzidine. Cells were washed twice with ice-cold PBS. The cell pellets were then re-suspended in ice-cold PBS $(45 \mu 1)$. The benzidine solution $(5 \mu 1)$ containing hydrogen peroxide (final concentration, $0.0012 \%$ ) was added at room temperature. Benzidine-positive cells were examined by light microscopy.

\section{Determination of hemoglobin}

The measurement of hemoglobin in K562 cells was as described [22]. Briefly, equal numbers of cells were washed with cold PBS and lysed for $20 \mathrm{~min}$ in lysis buffer $(0.2 \%$ Triton X-100 in $100 \mathrm{mM}$ potassium phosphate $\mathrm{pH} 7.8$ ). The lysates were centrifuged for 15 min at 1500 r.p.m. and $10 \mu \mathrm{l}$ of the supernatant was incubated with $2 \mathrm{ml}$ of the TMB solution (3,3',5,5'-tetramethylbenzidine; $5 \mathrm{mg} / \mathrm{ml}$ in glacial acetic acid) and $2 \mathrm{ml} \mathrm{30 \%} \mathrm{H}_{2} \mathrm{O}_{2}$ for $10 \mathrm{~min}$ at room temperature. Absorption was measured at $600 \mathrm{~nm}$. The hemoglobin content in $\mathrm{pg} / \mathrm{cell}$ was calculated from a hemoglobin standard curve and the data were shown as fold increases in comparison with the level of hemoglobin in mock-induced K562 cells. 


\section{Total RNA extraction and RT-PCR}

Total RNA from cultured cells was purified using the RNeasy Kit (Qiagen), according to the manufacturer's instructions. All RNA extracts were treated with RNase-free DNase to eliminate genomic DNA contamination. First, strand cDNAs were synthesized by reverse transcription conducted using a Qiagen Kit. Primers for the ALA-S2 amplification were 5'-GTC CCC TCG AGG AGT TGT GT-3' and 5'-ATC TTC CAT CAT CTG AGG GC-3'. Primers for the PBGD amplification were 5'-TAC TGC AGC GGC AGC AAC AGC AGG-3' and 5'-AGG CTT TCT AGG GTC TTC CCA ACA3'. Primers for the $\gamma$-globin amplification were 5'-ACT CGC TTC TGG AAC GTC TGA-3' and 5'-GTA TCT GGA GGA CAG GGC ACT-3'. Primers for the integrin $\gamma 3$ amplification were 5'-GCC TCT GGG CTC ACC TCG CTG-3' and 5'-CTG GGA RAG CTT CTC AGT CAT-3'. Primers for the $\beta$-actin amplification were 5'-GCA TCC CCC AAA GTT CAC AA-3' and 5'-AGG ACT GGG CCA TTC TCC TT-3'. PCR products were separated by agarose gel electrophoresis and visualized by ethidium bromide staining.

\section{Results}

Chemical induction of K562 cell erythroid differentiation

To examine the role of $\mathrm{N}-\mathrm{CoR}$ in erythropoiesis, we first established the conditions to induce K562 cell differentiation into erythroid lineage in vitro. We used hemin and Ara-C because both of them have been widely used for this purpose. We first conducted pilot experiments with different concentrations of inducing agents. Consistent with the data in the literature, we found that a 3-day treatment with $30 \mu \mathrm{M}$ hemin is sufficient to induce $\mathrm{K} 562$ cells to differentiate into an erythroid lineage, whereas higher levels of hemin (100 $\mu \mathrm{M}$ and above) led to increased cell death (data not shown). Similarly, we found that Ara-C at a concentration of $0.1 \mu \mathrm{g} / \mathrm{ml}$ effectively induced differentiation after a 6-day treatment. A typical result of a 3-day treatment with $30 \mu \mathrm{M}$ hemin and a 6-day treatment with $0.1 \mu \mathrm{g} / \mathrm{ml}$ Ara-C on K562 is shown in Figure 1A and 1D, respectively. A marked elevation in hemoglobin production was evident compared with the uninduced control cells by the appearance of dark-red cell pellets. As a more accurate measurement of erythroid differentiation, we also measured the level of hemoglobin 3 days after hemin or 6 days after Ara-C treatment, and the results are shown in Figure $1 \mathrm{~B}$ and $1 \mathrm{E}$. In addition, benzidine staining, which detects the pseudoperoxidase activity of hemoglobin [23], showed that the number of erythroid lineage cells increased from background levels (2-3\%) to more than $50-60 \%$ of the cell population after a 3-day hemin or a 6-day Ara-C treatment (Figure 1C and 1F). As reported previously [17], we also observed that hemin and Ara-C treatment inhibit

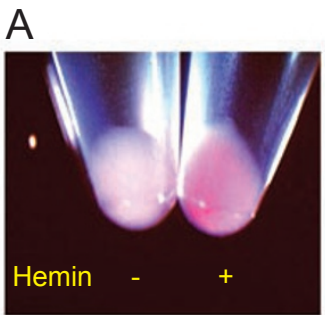

B
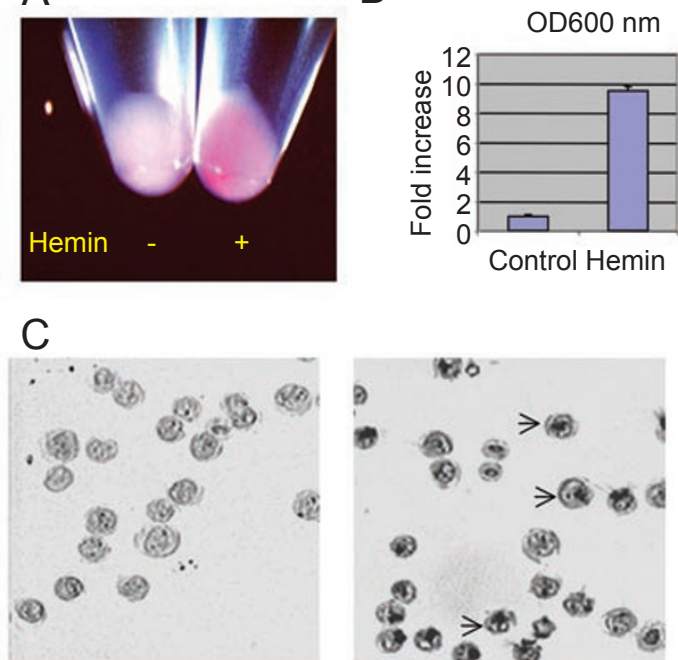

Control

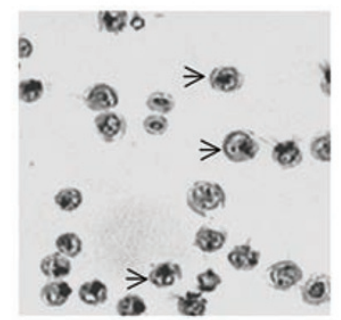

Hemin
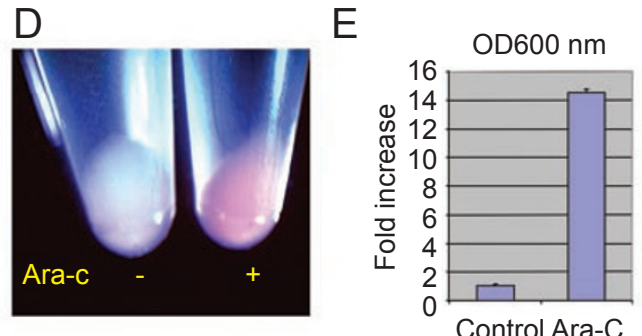

F
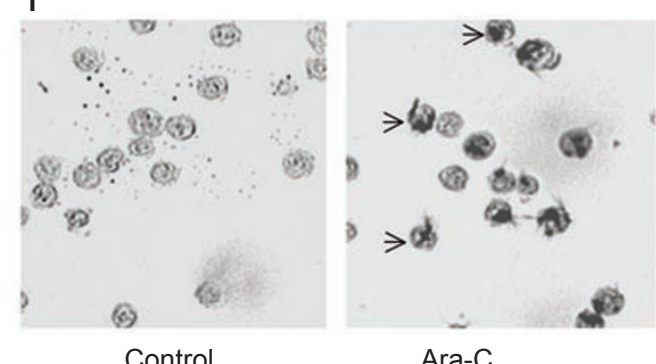

Figure 1 Both hemin and Ara-C induce K562 cells to differentiate into erythroid lineage cells. (A and D), K562 cells were induced for erythroid differentiation with $30 \mu \mathrm{M}$ hemin for 3 days (A) or $0.1 \mu \mathrm{g} / \mathrm{ml}$ Ara-C for 6 days (D). The cells were collected in $1.5 \mathrm{ml}$ tubes and photographed. The induced erythroid differentiation was evident by the pink-red cell pellets. (B and E); the amount of hemoglobin in the hemin- or Ara-C-induced K562 cells from (A) and (D) were determined as described in the Materials and Methods. The data are presented as the fold increase over the amount of hemoglobin in the mock-induced K562 cells. (C and F) K562 cells were induced with or without hemin for 3 days $(\mathbf{C})$ or Ara-C for 6 days $(\mathbf{F})$ and stained with benzidine. A few representative hemoglobin-positive cells are marked with arrows. 
cell proliferation (data not shown). These experiments established the conditions for hemin- and Ara-C-induced erythroid differentiation of K562 cells and thus allowed us to test the role of $\mathrm{N}-\mathrm{CoR}$ in this process.

\section{$N-C o R$ is essential for Ara-C but not hemin-induced ery- throid differentiation}

To examine the role of N-CoR in both hemin- and AraC-induced erythroid differentiation of K562 cells, we first used a specific siRNA to knock down the expression of $\mathrm{N}-\mathrm{CoR}$ in K562 cells. For this purpose, K562 cells were transfected with a control scramble siRNA or siN-CoR by electroporation. We found that transfection with $10 \mathrm{nM}$ siN-CoR reduced the level of N-CoR by more than $80 \% 2$ days after transfection (Figure 2A). This effect on N-CoR was sustained for up to a week and then the level of N-CoR recovered (data not shown).
A

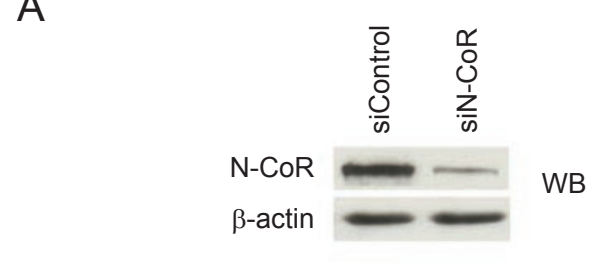

B

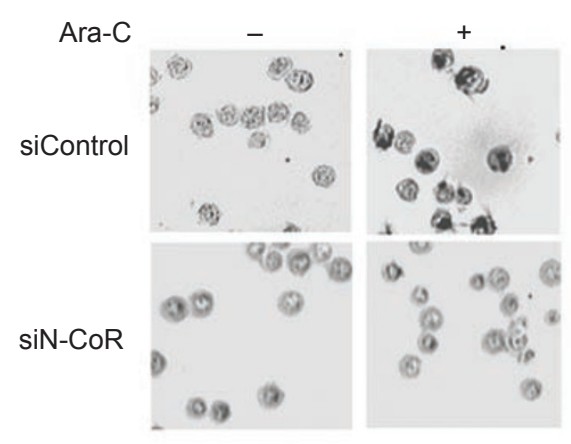

C

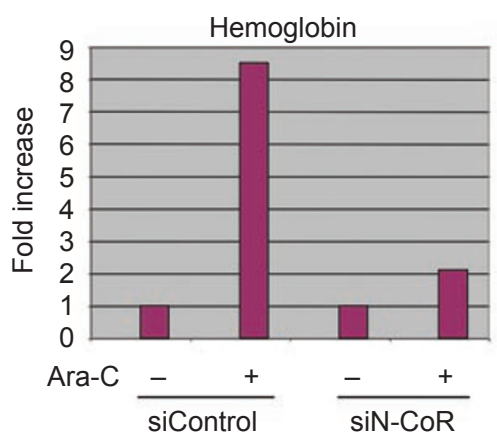

D

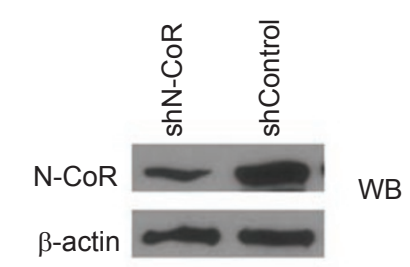

E

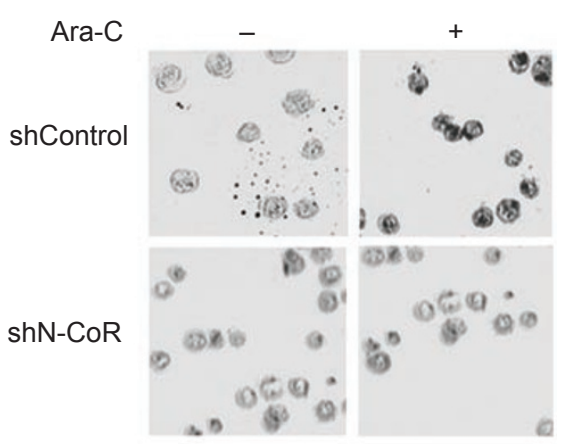

F

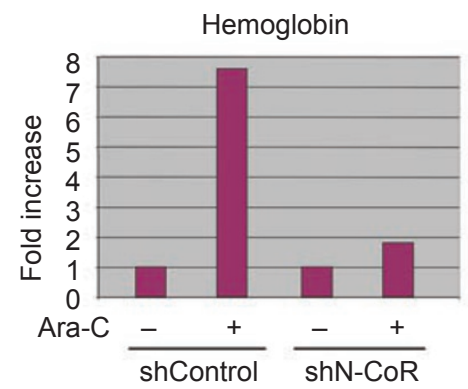

Figure 2 Knockdown of N-CoR transiently or stably impairs Ara-C-induced erythroid differentiation of K562 cells. (A) Western blotting using an N-CoR-specific antibody confirms the knockdown of N-CoR in K562 cells that are transfected with siN-CoR but not the siControl (scramble) for 3 days. Western blotting for $\beta$-actin serves as a loading control. (B) Knockdown of N-CoR in K562 cells by siRNA blocked Ara-C-induced erythroid differentiation, as revealed by benzidine staining. The K562 cells were transfected with control or siRNA against N-CoR for 3 days and then induced by Ara-C for 6 days before being processed for benzidine staining. (C) A representative result shows the effect of knockdown of N-CoR on the induction of hemoglobin synthesis by Ara-C. The result is based on duplicate samples from a single experiment (average value) and presented as the fold-induction. A similar effect was observed in two independent experiments. (D) Western blot analysis showed a significantly reduced level of N-CoR in K562 cells that stably express a human N-CoR-specific shRNA. As a control, the level of $\beta$-actin was similar in the stable shN-CoR and shControl (vector only) K562 cells. (E) Stable knockdown of N-CoR in K562 cells by shRNA also impairs Ara-C-induced erythroid differentiation, as revealed by benzidine staining. (F) Ara-C-induced hemoglobin synthesis was significantly reduced in the shN-CoR K562 cells. A similar result was observed in three independent experiments. 
A

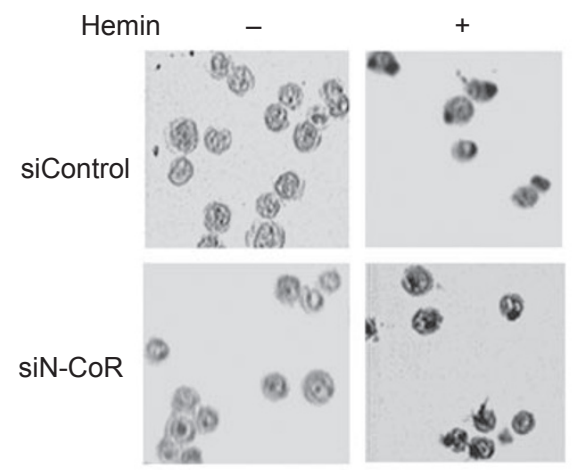

B

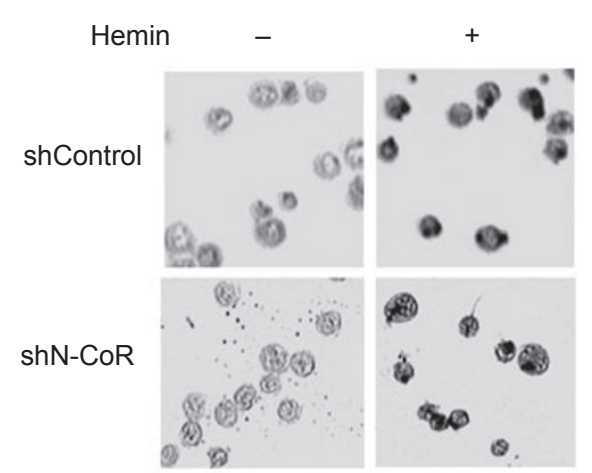

Figure 3 Knockdown of N-CoR in K562 cells has no effect on hemin-induced erythroid differentiation. (A) Benzidine staining reveals that the knockdown of $\mathrm{N}-\mathrm{CoR}$ by siN-CoR does not affect hemin-induced erythroid differentiation. K562 cells were transfected with control or siRNA against N-CoR for 3 days before induction with hemin $(30 \mu \mathrm{M})$ for 3 days and staining with benzidine. (B) The stable control and shN-CoR K562 cells were subjected to hemin treatment as in (A) and stained for hemoglobin-positive cells by benzidine. Note that the stable knockdown of N-CoR does not affect hemin-induced synthesis of hemoglobin.

We next tested the effect of knockdown of N-CoR on Ara-C- and hemin-induced differentiation of K562 cells. K562 cells were transfected with control siRNA or siN-CoR first, and 2 days after transfection the cells were induced for differentiation by $0.1 \mu \mathrm{g} / \mathrm{ml}$ Ara-C for 6 days and 30 $\mu \mathrm{M}$ hemin for 3 days. As shown in Figure 2B, we found that the knockdown of N-CoR substantially affected the Ara-C-induced differentiation as revealed by benzidine staining. By contrast, knockdown of N-CoR had no effect on hemin-induced differentiation (Figure 3A). Consistent with the results of benzidine staining (Figure 2B), we found that treatment with siN-CoR reduced the Ara-C-induced increase of hemoglobin from $\sim 8.5$-fold to only 2.1 -fold (Figure 2C). The same assay showed that treatment with siN-CoR did not affect the hemin-induced increase of hemoglobin (data not shown). Thus, these results suggest that N-CoR is required for Ara-C- but not hemin-induced K562 erythroid differentiation.

To verify the results derived from transient knockdown of N-CoR, we established a pool of stable K562 cells expressing either the vector or an shRNA against $\mathrm{N}-\mathrm{CoR}$. Western blot analysis confirmed the decrease in N-CoR in this pool of shN-CoR cells (Figure 2D). We then subjected the control and shN-CoR cells to hemin- and Ara-C-induced differentiation. Benzidine staining results in Figures $2 \mathrm{E}$ and $3 \mathrm{~B}$ show that the shN-CoR K562 cells were again defective in Ara-C- but not hemin-induced differentiation. Again, the defect of the shN-CoR K562 cells in Ara-C-induced erythroid differentiation was confirmed by direct measurement of the hemoglobin level (Figure 2F). Taken together, these results reveal an essential role for N-CoR in Ara-C- but not hemin-induced erythroid differentiation.
Ara-C induces the expression of a key enzyme required for heme synthesis

Hemoglobinization is one of the hallmarks of erythroid differentiation and requires the coordinated synthesis of heme and globin [24]. In principle, hemin can promote K562 cell hemoglobinization by enhancing the synthesis of the fetal and embryonic globins and bypassing the requirement for heme synthesis, whereas Ara-C has to induce the synthesis of both heme and globin. Given that the knockdown of N-CoR only impairs Ara-C- but not hemin-induced $\mathrm{K} 562$ cell differentiation, we reason that $\mathrm{N}-\mathrm{CoR}$ is probably required for the synthesis of heme. As illustrated in Figure 4A, the synthesis of heme involves eight steps. In erythrocytes, the enzyme ALA-S2 controls the rate-limiting step of the heme synthesis from glycine to 5-aminolevulinic acid [24]. Previous studies have shown that ALA-S2 is often induced during erythroid differentiation [25-28], we thus analyzed whether Ara-C also induces the expression of ALA-S2 in K562 cells. K562 cells were subjected to Ara-C- and hemin-induced erythroid differentiation for 3 days and the levels of ALA-S2 mRNA were then determined by RT-PCR. The results in Figure 4B show that ALA-S2 was induced by three- to four-fold by Ara-C. Although hemin was reported to induce the expression of ALA-S2 in K562 cells, with a 7-day treatment with hemin, its effect on ALA-S2 expression was weak in comparison with Ara-C under our experimental conditions. We also analyzed the level of the fetal $\gamma$-globin mRNA, and the result in Figure 4C shows that the expression of $\gamma$-globin was also induced by about three- to four-fold after a 3-day treatment with Ara-C, which is consistent with a previous study [17]. It should be pointed out that the induction of 
A

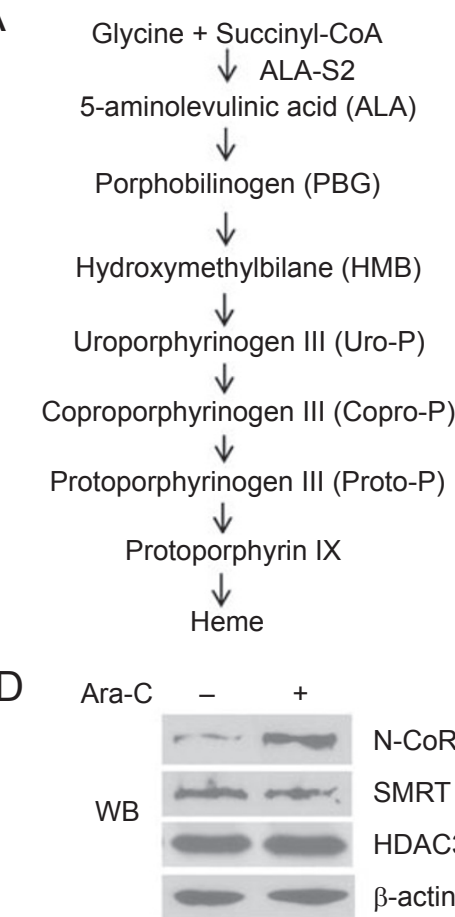

B

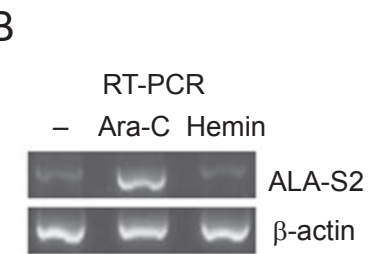

C RT-PCR

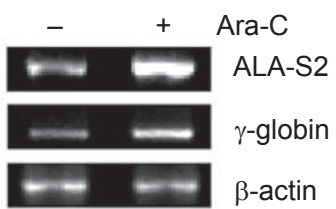

E

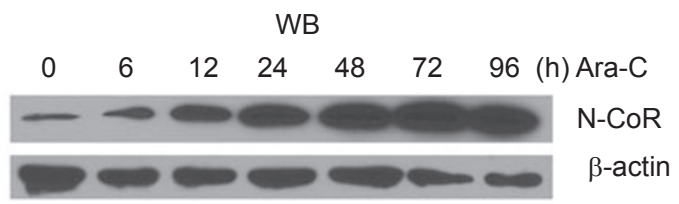

F RT-PCR

\begin{tabular}{|c|c|c|c|c|c|c|c|}
\hline 0 & 6 & 12 & 24 & 48 & 72 & 96( & (h) Ara-C \\
\hline 0 & - & 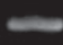 & $=$ & 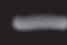 & حس & 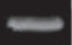 & $\mathrm{N}-\mathrm{CoR}$ \\
\hline & 4 & & 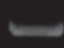 & ص & G & 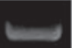 & ALA-S2 \\
\hline$\theta$ & 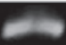 & & & & 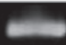 & 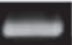 & GAPDH \\
\hline
\end{tabular}

Figure 4 Ara-C induces the expression of key enzymes that are required for heme synthesis as well as N-CoR. (A) The diagram illustrates the pathway for synthesis of heme in erythrocytes. Also the enzyme ALA-S2 for the rate-limiting step of the pathway is shown. (B) In comparison with hemin, Ara-C strongly induces the expression of ALA-S2 in K562 cells. K562 cells were treated with $30 \mu \mathrm{M}$ hemin for 3 days or $0.1 \mu \mathrm{g} / \mathrm{ml}$ Ara-C for 6 days and total RNA was prepared from these cells. An equal amount of total RNA was analyzed for the levels of ALA-S2 by RT-PCR. (C) Ara-C treatment also induced the expression of $\gamma$-globin. The K562 cells were treated with Ara-C and analyzed by RT-PCR as in (B). (D) Western blot analysis reveals that Ara-C treatment also induces the expression of N-CoR. The K562 cells were treated with $0.1 \mu \mathrm{g} / \mathrm{ml}$ Ara-C for 6 days. The whole-cell extracts were prepared and analyzed by western blotting using antibodies. Note that under the same conditions SMRT and HDAC3 were not induced by Ara-C. (E) Ara-C-induced erythroid differentiation is associated with a gradually increased level of N-CoR protein. K562 cells were treated with Ara-C for various times as indicated and collected for analysis of the levels of N-CoR protein by western blotting. As a control, the levels of $\beta$-actin were also analyzed by western blotting. (F) Ara-C did not induce the increased transcription of N-CoR mRNA. K562 cells were treated as above and the levels of N-CoR mRNA and ALA-S2 were determined by semi-quantitative RT-PCR.

ALA-S2 by Ara-C is both dose and time dependent. At a high dose $(1 \mu \mathrm{g} / \mathrm{ml})$ of Ara-C, ALA-S2 mRNA increased dramatically ( $>10$-fold) after 3 days of induction, whereas a significant increase of ALA-S2 mRNA was only observed after a longer period of induction ( 6 days) when a low dose of Ara-C (20 ng/ml) was used (data not shown).

Treatment with Ara-C increases the amount of $\mathrm{N}-\mathrm{CoR}$ protein through a post-transcriptional mechanism

In the process of analyzing Ara-C-induced K562 cell differentiation, we found that the amount of $\mathrm{N}-\mathrm{CoR}$ protein increased significantly during Ara-C-induced differentiation. A representative result is shown in Figure 4D. In this experiment, whole-cell extracts were prepared from the K562 cells that were mock induced or induced with Ara-
C $(0.1 \mu \mathrm{g} / \mathrm{ml})$ for 3 days and subjected to western blot analysis using antibodies. We estimate that treatment with Ara-C increased the amount of N-CoR protein by about three times. This effect is specific, as neither the level of the highly related SMRT protein nor the level of HDAC3 changed during this process (Figure 4D).

To characterize the induction of N-CoR by Ara-C in more detail, we performed time course experiments and a representative result is shown in Figure 4E. We found that the level of $\mathrm{N}-\mathrm{CoR}$ protein increased by $\sim 2$-fold after a 6-h treatment with Ara-C and increased further along in the 96-h Ara-C treatment process ( $\sim$ six- to eight-fold). Interestingly, RT-PCR analysis revealed a constant level of N-CoR mRNA during the same time course (Figure 4F). Thus, Ara-C apparently increases the amount of N-CoR 
A
A

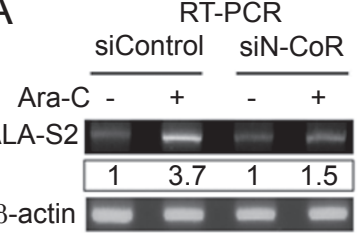

B

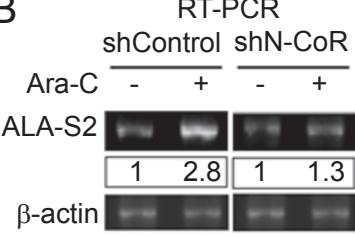

Figure $5 \mathrm{~N}-\mathrm{CoR}$ is required for the optimal induction of the enzyme ALA-S2 by Ara-C. (A) Knockdown of N-CoR by siRNA impairs Ara-C-induced ALA-S2 expression. K562 cells were transfected with a control or siRNA against N-CoR for 6 days to knock down $\mathrm{N}-\mathrm{CoR}$. The K562 cells were then induced by Ara-C for 3 days. The total RNA was prepared and analyzed for the levels of ALA-S2 by RT-PCR. (B) In the shN-CoR K562 cells, the induction of ALA-S2 by Ara-C was also impaired. The control and shN-CoR K562 cells were treated with Ara-C for 6 days and the levels of ALA-S2 were analyzed by RT-PCR. The RT-PCR results were quantified using a densitometer and the results are the average value of two independent experiments. through a post-transcriptional mechanism. The result that $\mathrm{N}-\mathrm{CoR}$ is induced by Ara-C supports a role for $\mathrm{N}-\mathrm{CoR}$ in ARA-C-induced K562 erythroid differentiation.

\section{$N-C o R$ is required for the induction of key enzymes involved} in heme synthesis by Ara-C

Given that N-CoR is required for Ara-C-induced K562 erythroid differentiation (Figure 2), we next examined whether $\mathrm{N}-\mathrm{CoR}$ is required for the Ara-C-induced expression of ALA-S2. We used siRNA to knock down N-CoR in $\mathrm{K} 562$ cells first and then subjected the cells to Ara-C treatment. The results in Figure 5A show that the induction of ALA-S2 by Ara-C was significantly diminished in the K562 cells that were treated with siN-CoR but not with the control siRNA. The knockdown of N-CoR by siN-CoR was confirmed by western blot analysis (data not shown). Thus, this result indicates that N-CoR is essential for Ara-
A

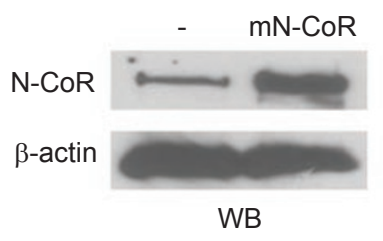

C

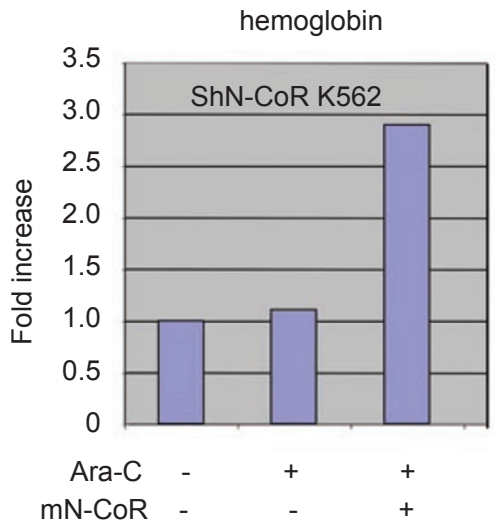

B

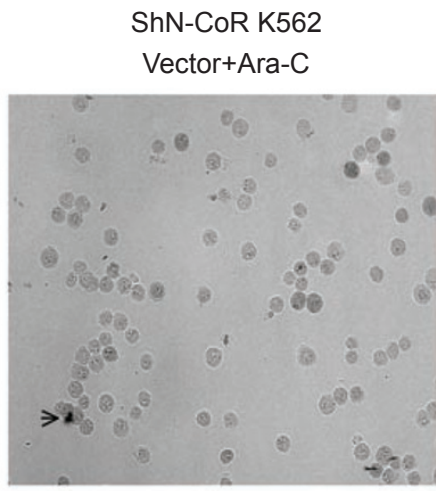

$\mathrm{mN}-\mathrm{CoR}+$ Ara-C

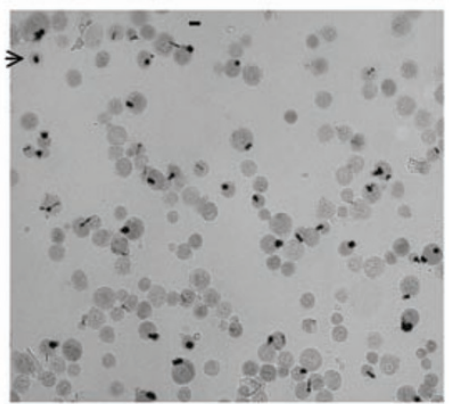

Figure 6 Partial rescue of an erythroid differentiation defect in the shN-CoR K562 cells by ectopic expression of mouse N-CoR. (A) shN-CoR K562 cells were transfected with an expression construct for mouse N-CoR. Two days after transfection, the level of $\mathrm{N}-\mathrm{CoR}$ was determined by western blotting. (B) The shN-CoR cells were transfected with mN-CoR and subjected to Ara-C treatment for 6 days. The erythroid lineage cells were then revealed by benzidine staining. The low magnification data are shown in order to highlight the sporadic hemoglobin-positive cells in the absence of ectopic N-CoR expression. The low efficiency of rescue by $\mathrm{mN}-\mathrm{CoR}$ is most likely the result of low plasmid transfection efficiency (15-20\%) for K562 cells. (C) The shN-CoR cells were treated as in (B) and the levels of hemoglobin were determined and presented as the fold increase over the level of hemoglobin in the mock-induced shN-CoR K562 cells. The results were the average value of two representative experiments and were later confirmed by an independent experiment. 
C-induced expression of ALA-S2.

To substantiate the role of N-CoR in the induction of ALA-S2, we also analyzed the mRNA levels of the ALA$\mathrm{S} 2$ before and after Ara-C induction in the shRNA control and shN-CoR K562 cells. As shown in Figure 5B, Ara-C treatment induced the expression of ALA-S2 in the shRNA control K562 cells. However, Ara-C treatment failed to induce the expression of ALA-S2 in the shN-CoR K562 cells. Altogether, these results demonstrate that $\mathrm{N}-\mathrm{CoR}$ is essential for Ara-C-induced expression of a key enzyme that is required for heme synthesis, thus providing an explanation for why N-CoR is required for Ara-C-induced erythroid differentiation.

Expression of mouse $N$-CoR rescues the differentiation defect of the N-CoR knockdown K562 cells

To confirm whether the defect in erythroid differentiation that we observed in the stable shN-CoR K562 cells is a consequence of N-CoR knockdown, we performed a rescue experiment by expressing mouse $\mathrm{N}-\mathrm{CoR}$, whose expression is not affected by the shRNA that is specific to the human N-CoR. As shown in Figure 6A, transfection of a mouse N-CoR expression construct (pCMX-mN-CoR) led to increased levels of N-CoR in the stable shN-CoR K562 cells, as shown by western blot analysis using an antibody that recognizes both human and mouse N-CoR. Immunostaining revealed that $\sim 20-25 \%$ cells were transfected with mouse N-CoR (data not shown). Importantly, when the shN-CoR K562 cells that were transfected with mN-CoR were subjected to erythroid differentiation by Ara-C treatment, a significant increase of benzidine positively stained cells was detected (from $\sim 3 \%$ to $16.4 \%$; Figure $6 \mathrm{~B}$ ). As a control, the shN-CoR K562 cells transfected with the empty pCMX vector did not significantly increase the number of positive benzidine stained cells. We also directly measured the levels of hemoglobin and the results in Figure 6C show
A
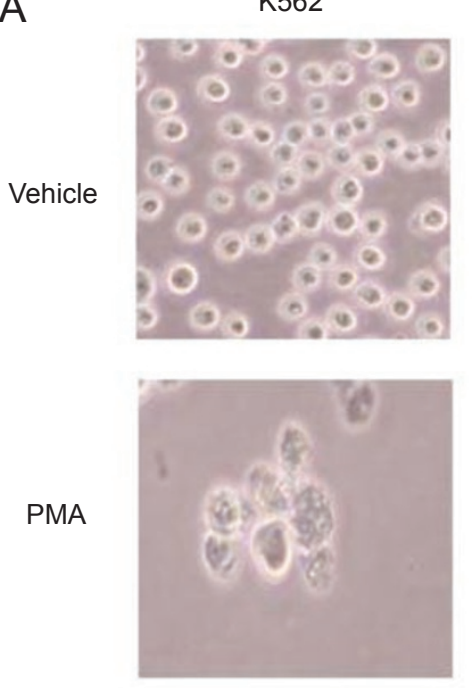

$\operatorname{shN}-\mathrm{CoR}$
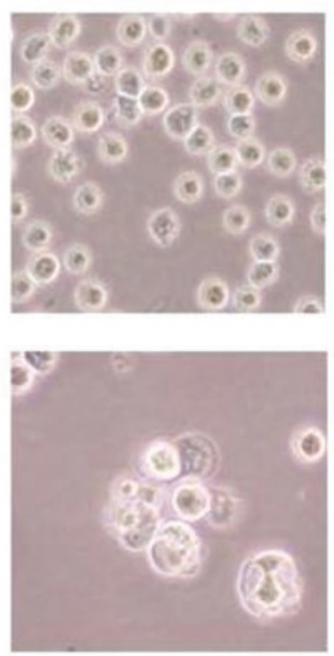

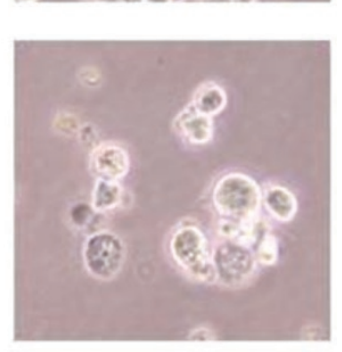

shControl

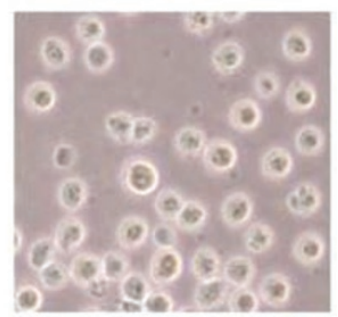

B

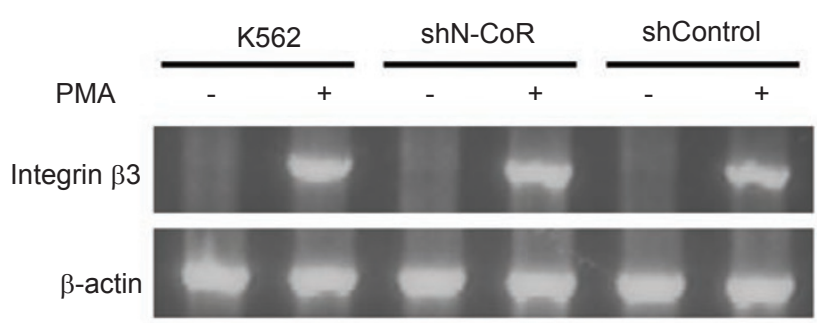

Figure $7 \mathrm{~N}-\mathrm{CoR}$ is not required for the PMA-induced differentiation of K562 cells to megakaryocytes. (A) Different K562 cells were treated with $50 \mathrm{mM}$ PMA for 4 days to induce differentiation to megakaryocytes as described in the Materials and Methods. The results show that this differentiation, which is evident as a morphological change, was not dependent on N-CoR. (B) The PMA-induced differentiation to megakaryocytes was confirmed by the induction of the megakaryocyte marker integrin $\beta$ III/CD61 by RT-PCR analysis. 
an approximately two-fold increase over the vector-transfected cells. Given that in this experiment only $\sim 20-25 \%$ of the cells were actually expressing $\mathrm{mN}-\mathrm{CoR}$, this result suggests that most of the mN-CoR-transfected cells could be induced toward the erythroid lineage by Ara-C. We thus conclude that the expression of $\mathrm{mN}-\mathrm{CoR}$ is able to rescue the erythroid differentiation defect associated with the shN-CoR K562 cells.

Knockdown of $N$-CoR had no effect on the differentiation of K562 cells to megakaryocytes

Many lines of evidence demonstrate that erythroids and megakaryocytes are closely related to each other and share a common precursor cell [29]. It is well known that K562 cells can be induced to either erythroid or megakaryocytic linages, depending on the inducing agents used $[14,15$, 30]. For example, K562 can be induced by PMA to differentiate into the megakaryocytic lineage [31]. Since the knockdown of N-CoR impairs Ara-C-induced K562 erythroid differentiation, we wished to test whether $\mathrm{N}-\mathrm{CoR}$ is specifically involved in erythroid differentiation or also participates in the megakaryocytic pathway. As shown in Figure 7A, PMA treatment successfully induced K562 megakaryocytic differentiation, which is characterized by the inhibition of cell proliferation, increased cell size and megakaryocytic morphology (multinuclei). As a molecular marker for megakaryocytic differentiation [32], PMA treatment drastically increased the mRNA level of integrin $\beta 3$ (also known as antigen CD61; Figure 7B). However, compared with either the K562 parental cells or the stable control shRNA K562 cells, knockdown of N-CoR had no effect on megakaryocytic differentiation as shown by the PMA-induced morphological changes (Figure 7A) as well as the induction of integrin- $\beta 3$ mRNA (Figure 7B). Thus, consistent with the normal differentiation of megakaryocytes in N-CoR-null mice [5], our in vitro results indicate that $\mathrm{N}-\mathrm{CoR}$ is not required for the differentiation of K562 cells to megakaryocytes.

\section{Discussion}

In this report we make use of the human erythroleukemic $\mathrm{K} 562$ cell line to investigate the role of $\mathrm{N}-\mathrm{CoR}$ in erythroid and megakaryocyte differentiation. We show that N-CoR is required for Ara-C- but not hemin-induced differentiation to the erythroid lineage. In addition, we show that $\mathrm{N}-\mathrm{CoR}$ is not required for PMA-induced differentiation of K562 cells into megakaryocytic cells. Thus, this in vitro study is in agreement with the phenotypes of N-CoR-/- mice that have defects in definitive erythropoiesis but have a normal number of megakaryocytes [5]. Consistent with a role for $\mathrm{N}-\mathrm{CoR}$ in erythropoiesis, we find that $\mathrm{N}-\mathrm{CoR}$ is induced during Ara-C-induced erythroid differentiation. Moreover, we show that $\mathrm{N}-\mathrm{CoR}$ is required for the Ara-C-induced expression of ALA-S2, a key enzyme that is required for heme synthesis.

Both Ara-C and hemin can induce K562 cell differentiation into erythroid cells. Although the way that these agents induce K562 cell differentiation remains to be fully elucidated, they probably do so through different pathways. As shown by Tahara et al. [33, 34], heme has a dual role during K562 cell erythroid differentiation. In addition to serving as a direct prosthetic substrate for globin, it also upregulates globin chain synthesis by repressing the transcriptional repressor Bach1, and subsequently enhances globin gene transcription. Furthermore, heme also enhances globin expression at the level of translation through its ability to regulate eIF2 $\alpha$ kinase activity [35]. Based on our observation that N-CoR is required for Ara-C- but not hemin-induced erythroid differentiation, we turn our attention to the roles of N-CoR in heme biosynthesis. There are eight steps in the heme biosynthesis pathway and each step is catalyzed by a unique enzyme. The enzyme 5 -aminolevulinic acid synthase ALA-S2 is the first enzyme in the heme biosynthesis pathway and is responsible for the differences in regulation and rates of heme synthesis in erythroid and non-erythroid cells [24]. It was previously shown that the level of ALA-S2 mRNA increases in the Ara-C-induced human myelogenous leukemia cell line YN-12 [26]. We show in this study that Ara-C also induces the expression of ALA-S2 mRNA in K562 cells. Thus, Ara-C-induced K562 cell differentiation is associated with the induction of a key enzyme that is involved in heme synthesis. Importantly, we find that $\mathrm{N}-\mathrm{CoR}$ is required for Ara-C-induced expression of ALA-S2, since the knockdown of N-CoR either transiently by siRNA or permanently by shRNA impairs the induction of the enzyme. Thus, our results provide the first evidence that $\mathrm{N}-\mathrm{CoR}$ has a role in regulating the expression of a key enzyme that is involved in the heme biosynthesis pathway.

Interestingly, $\mathrm{N}-\mathrm{CoR}$ is required for Ara-C-induced erythroid differentiation and its own level in K562 cells is also induced by Ara-C (Figure 4). We show that upon treatment with Ara-C, the amount of N-CoR protein in K562 cells gradually increases. Ara-C appears to affect the level of N-CoR via a post-transcriptional mechanism, as Ara-C treatment has no effect on the levels of N-CoR mRNA(Figure 4). The fact that $\mathrm{N}-\mathrm{CoR}$ is induced by Ara-C supports a role for $\mathrm{N}-\mathrm{CoR}$ in Ara-C-induced erythroid differentiation. Future work is needed to determine whether Ara-C increases the level of $\mathrm{N}-\mathrm{CoR}$ by enhancing the translation of N-CoR mRNA and/or protein stability.

At this stage, how $\mathrm{N}-\mathrm{CoR}$ regulates the expression of ALA-S2 is not known. It is well established that N-CoR 
and its related protein SMRT [36, 37] form stable protein complexes with HDAC3 and other proteins and function as co-repressors for many transcriptional factors [1, 38-41]. In rare cases, N-CoR and HDAC3 have also been implicated in the transcriptional activation process [5], although the underlying mechanism is unknown. Given its well-characterized co-repressor function, it is possible that N-CoR indirectly regulates the expression of ALA-S2 by suppressing the expression or function of a transcription factor that represses ALA-S2. In this regard, the Id (inhibitor of DNA binding) family of helix-loop-helix repressors is known to enhance cell proliferation and inhibit cellular differentiation in many cell types, including erythroid lineage cells, and thus could be potential candidates for genes that are repressed by N-CoR $[42,43]$. However, we have not observed any effect of knockdown of N-CoR on the expression of Id1 and Id2 in K562 cells (data not shown), although we have yet to analyze the expression of Id 3 and Id4. Alternatively, N-CoR could have an essential role in modulating the activity of the transcription factors that are important for erythroid differentiation. An increasing number of transcription factors have been shown to have critical roles in erythroid differentiation, including GATA family members, EKLF, c-myb, NF-E2 and TAL1/SCL [44-46]. GATA-1, GATA-2 and GATA-3 transcription factors are important in regulating gene expression during hematopoiesis and in determining hematopoietic cell lineages. For instance, GATA-2 is critical in the development of hematopoietic stem cells $[47,48]$. Loss of GATA-1 blocks erythroid maturation $[48,49]$, whereas GATA-3 is required for the production of T-lymphocytes [50]. Furthermore, the ALAS2 immediate promoter contains binding sites for GATA1 and NF-E2 [51]. We have tested the potential interaction of N-CoR with GATA-1, c-myb, EKLF and TAL1/SCL using in vitro GST pulldown and co-immunoprecipitation, and we have so far failed to detect any significant interaction between N-CoR and these factors. However, it remains possible that $\mathrm{N}-\mathrm{CoR}$ could interact indirectly with these transcription factors or directly with the transcription factors we have yet to analyze. Nevertheless, the findings in this study that N-CoR is required for Ara-C-induced K562 erythroid differentiation and the expression of ALA-S2 open avenues for future investigation of the underlying molecular mechanism.

\section{Acknowledgments}

We thank Dr Chan (Department of Pharmacology, Baylor College of Medicine) for providing K562 cells and Dr Ho-Geun Yoon for discussion. This work was supported by the NIH Grant DK58679 to J Wong.

\section{References}

1 Jepsen K, Rosenfeld MG. Biological roles and mechanistic actions of co-repressor complexes. J Cell Sci 2002; 115:689-698.

2 Levine M, Tjian R. Transcription regulation and animal diversity. Nature 2003; 424:147-151.

3 Horlein AJ, Naar AM, Heinzel T, et al. Ligand-independent repression by the thyroid hormone receptor mediated by a nuclear receptor co-repressor. Nature 1995; 377:397-404.

4 Chen JD, Evans RM. A transcriptional co-repressor that interacts with nuclear hormone receptors. Nature 1995; 377:454-457.

5 Jepsen K, Hermanson O, Onami TM, et al. Combinatorial roles of the nuclear receptor corepressor in transcription and development. Cell 2000; 102:753-763.

6 Dhordain P, Albagli O, Lin RJ, et al. Corepressor SMRT binds the BTB/POZ repressing domain of the LAZ3/BCL6 oncoprotein. Proc Natl Acad Sci USA 1997; 94:10762-10767.

7 Kao HY, Ordentlich P, Koyano-Nakagawa N, et al. A histone deacetylase corepressor complex regulates the Notch signal transduction pathway. Genes Dev 1998; 12:2269-2277.

8 Yoon HG, Chan DW, Reynolds AB, et al. N-CoR mediates DNA methylation-dependent repression through a methyl $\mathrm{CpG}$ binding protein Kaiso. Mol Cell 2003; 12:723-734.

9 Tomita A, Buchholz DR, Shi YB. Recruitment of N-CoR/SMRTTBLR1 corepressor complex by unliganded thyroid hormone receptor for gene repression during frog development. Mol Cell Biol 2004; 24:3337-3346.

10 Rosenfeld MG, Lunyak VV, Glass CK. Sensors and signals: a coactivator/corepressor/epigenetic code for integrating signaldependent programs of transcriptional response. Genes Dev 2006; 20:1405-1428.

11 Lozzio CB, Lozzio BB. Human chronic myelogenous leukemia cell-line with positive Philadelphia chromosome. Blood 1975; 45:321-334

12 Rutherford TR, Clegg JB, Weatherall DJ. K562 human leukaemic cells synthesise embryonic haemoglobin in response to haemin. Nature 1979; 280:164-165.

13 Housset M, Daniel MT, Degos L. Small doses of ARA-C in the treatment of acute myeloid leukaemia: differentiation of myeloid leukaemia cells? Br J Haematol 1982; 51:125-129.

14 Watanabe T, Mitchell T, Sariban E, et al. Effects of 1-beta-Darabinofuranosylcytosine and phorbol ester on differentiation of human K562 erythroleukemia cells. Mol Pharmacol 1985; 27:683-688.

15 del Senno L, Conconi F, Barbieri R, et al. Human leukemic K562 cells: differential effects of 5-azacytidine on DNA methylation of epsilon-, gamma-globin and 7SL RNA genes. Boll Soc Ital Biol Sper 1984; 60:1613-1619.

16 Fibach E, Kollia P, Schechter AN, et al. Hemin-induced acceleration of hemoglobin production in immature cultured erythroid cells: preferential enhancement of fetal hemoglobin. Blood 1995; 85:2967-2974.

17 Takagaki K, Katsuma S, Horio T, et al. cDNA microarray analysis of altered gene expression in Ara-C-treated leukemia cells. Biochem Biophys Res Commun 2003; 309:351-358.

18 Kufe DW, Major PP, Egan EM, Beardsley GP. Correlation of cytotoxicity with incorporation of ara-C into DNA. J Biol Chem 1980; 255:8997-8900. 
19 Kufe DW, Major PP. Studies on the mechanism of action of cytosine arabinoside. Med Pediatr Oncol 1982; 10 Suppl 1:4967.

20 Zhang D, Yoon HG, Wong J. JMJD2A is a novel N-CoR-interacting protein and is involved in repression of the human transcription factor achaete scute-like homologue 2 (ASCL2/Hash2). Mol Cell Biol 2005; 25:6404-6414.

21 Ueki N, Zhang L, Hayman MJ. Ski negatively regulates erythroid differentiation through its interaction with GATA1. Mol Cell Biol 2004; 24:10118-10125.

22 Woessmann W, Zwanzger D, Borkhardt A. ERK signaling pathway is differentially involved in erythroid differentiation of K562 cells depending on time and the inducing agent. Cell Biol Int 2004; 28:403-410.

23 Kaiho S, Mizuno K. Sensitive assay systems for detection of hemoglobin with 2,7-diaminofluorene: histochemistry and colorimetry for erythrodifferentiation. Anal Biochem 1985; 149:117-120.

24 Ponka P. Cell biology of heme. Am J Med Sci 1999; 318:241256.

25 Taketani S, Furukawa T, Furuyama K. Expression of coproporphyrinogen oxidase and synthesis of hemoglobin in human erythroleukemia K562 cells. Eur J Biochem 2001; 268:17051711.

26 Nagai T, Harigae H, Furuyama K, et al. 5-Aminolevulinate synthase expression and hemoglobin synthesis in a human myelogenous leukemia cell line. J Biochem (Tokyo) 1997; 121:487-495.

27 Zoller H, Decristoforo C, Weiss G. Erythroid 5-aminolevulinate synthase, ferrochelatase and DMT1 expression in erythroid progenitors: differential pathways for erythropoietin and irondependent regulation. Br J Haematol 2002; 118:619-626.

28 Chenais B, Molle I, Trentesaux C, et al. Time-course of butyric acid-induced differentiation in human K562 leukemic cell line: rapid increase in gamma-globin, porphobilinogen deaminase and NF-E2 mRNA levels. Leukemia 1997; 11:1575-1579.

29 McDonald TP, Sullivan PS. Megakaryocytic and erythrocytic cell lines share a common precursor cell. Exp Hematol 1993; 21:1316-1320.

30 Reading CL, Hickey CM, Yong WB. Analysis of cell surface glycoprotein changes related to hematopoietic differentiation. J Cell Biochem 1988; 37:21-36.

31 Butler TM, Ziemiecki A, Friis RR. Megakaryocytic differentiation of K562 cells is associated with changes in the cytoskeletal organization and the pattern of chromatographically distinct forms of phosphotyrosyl-specific protein phosphatases. Cancer Res 1990; 50:6323-6329.

32 Alitalo R. Induced differentiation of K562 leukemia cells: a model for studies of gene expression in early megakaryoblasts. Leuk Res 1990; 14:501-514.

33 Tahara T, Sun J, Nakanishi K, et al. Heme positively regulates the expression of beta-globin at the locus control region via the transcriptional factor Bach1 in erythroid cells. J Biol Chem 2004; 279:5480-5487.

34 Tahara T, Sun J, Igarashi K, et al. Heme-dependent up-regulation of the alpha-globin gene expression by transcriptional repressor Bach1 in erythroid cells. Biochem Biophys Res Commun 2004;
324:77-85.

35 Han AP, Fleming MD, Chen JJ. Heme-regulated eIF2alpha kinase modifies the phenotypic severity of murine models of erythropoietic protoporphyria and beta-thalassemia. J Clin Invest 2005; 115:1562-1570.

36 Park EJ, Schroen DJ, Yang M, et al. SMRTe, a silencing mediator for retinoid and thyroid hormone receptors-extended isoform that is more related to the nuclear receptor corepressor. Proceedings of the National Academy of Sciences of the United States of America 1999; 96:3519-3524.

37 Ordentlich P, Downes M, Xie W, et al. Unique forms of human and mouse nuclear receptor corepressor SMRT. Proceedings of the National Academy of Sciences of the United States of America 1999; 96:2639-2644.

38 Guenther MG, Lane WS, Fischle W, et al. A core SMRT corepressor complex containing HDAC3 and TBL1, a WD40-repeat protein linked to deafness. Genes Dev 2000; 14:1048-1057.

39 Li J, Wang J, Nawaz Z, et al. Both corepressor proteins SMRT and $\mathrm{N}-\mathrm{CoR}$ exist in large protein complexes containing HDAC3. EMBO J 2000; 19:4342-4350.

40 Zhang J, Kalkum M, Chait BT, et al. The N-CoR-HDAC3 nuclear receptor corepressor complex inhibits the JNK pathway through the integral subunit GPS2. Mol Cell 2002; 9:611-623.

41 Yoon HG, Chan DW, Huang ZQ, et al. Purification and functional characterization of the human N-CoR complex: the roles of HDAC3, TBL1 and TBLR1. EMBO J 2003; 22:1336-1346.

42 Benezra R, Davis RL, Lockshon D, et al. The protein Id: a negative regulator of helix-loop-helix DNA binding proteins. Cell 1990; 61:49-59.

43 Lister J, Forrester WC, Baron MH. Inhibition of an erythroid differentiation switch by the helix-loop-helix protein Id1. J Biol Chem 1995; 270:17939-17946.

44 Cantor AB, Orkin SH. Transcriptional regulation of erythropoiesis: an affair involving multiple partners. Oncogene 2002; 21:3368-3376.

45 Lecuyer E, Hoang T. SCL: from the origin of hematopoiesis to stem cells and leukemia. Exp Hematol 2004; 32:11-24.

46 Ohneda K, Yamamoto M. Roles of hematopoietic transcription factors GATA-1 and GATA-2 in the development of red blood cell lineage. Acta Haematol 2002; 108:237-245.

47 Tsai FY, Orkin SH. Transcription factor GATA-2 is required for proliferation/survival of early hematopoietic cells and mast cell formation, but not for erythroid and myeloid terminal differentiation. Blood 1997; 89:3636-3643.

48 Fujiwara Y, Chang AN, Williams AM, et al. Functional overlap of GATA-1 and GATA-2 in primitive hematopoietic development. Blood 2004; 103:583-585.

49 Pevny L, Lin CS, D'Agati V, et al. Development of hematopoietic cells lacking transcription factor GATA-1. Development 1995; 121:163-172.

50 Oosterwegel M, Timmerman J, Leiden J, et al. Expression of GATA-3 during lymphocyte differentiation and mouse embryogenesis. Dev Immunol 1992; 3:1-11.

51 Cox TC, Bawden MJ, Martin A, et al. Human erythroid 5-aminolevulinate synthase: promoter analysis and identification of an iron-responsive element in the mRNA. EMBO J 1991; 10:18911902. 